\title{
Calidad del informe de alta hospitalaria realizado por médicos internos residentes de especialidades quirúrgicas
}

\author{
Jenaro A. Fernández-Valencia, Artur Conesa, Moisés Ríos, Marta Catalán, Xavier Morales, Josep Riba
}

Introducción. Los médicos internos residentes (MIR) deben adquirir competencia en la correcta redacción del informe de alta hospitalaria (IAH). Este documento debe contener un conjunto de datos mínimos y ha de ser comprensible para el paciente, su médico de familia u otros profesionales. El proceso de adquisición de esta competencia ha sido poco estudiado y la calidad de los IAH firmados por MIR no se ha evaluado previamente.

Sujetos y métodos. El presente estudio ha evaluado la calidad de $124 \mathrm{IAH}$ de urgencias comparando aquellos firmados por especialistas, firmados únicamente por MIR o firmados por MIR y especialista, en las especialidades de cirugía ortopédica y traumatología y de cirugía general.

Resultados. El resultado ha sido más favorable que el de publicaciones previas, con una puntuación superior a 7 sobre 10 en el $94,4 \%$ de los casos. Además, los IAH realizados por residentes de primer año y supervisados por especialistas han sido los informes con mejor puntuación, con diferencias estadísticamente significativas $(p=0,003)$. No se observaron diferencias estadísticamente significativas entre los informes redactados por la mañana respecto de los redactados por la madrugada $(p=0,6)$. En cuanto a los contenidos, un 12,9\% de los informes presentaba siglas de difícil comprensión.

Conclusión. Nuestro estudio objetiva el efecto beneficioso de la supervisión del IAH en el primer año de residencia, al igual que se identifica un objetivo de mejora consistente en minimizar el uso de siglas médicas.

Palabras clave. Educación médica de posgrado. Informe de alta hospitalaria. MIR.

\section{Quality of hospital discharge report conducted by surgical resident physicians}

Introduction. Resident intern physicians (MIR) must become competent in writing correct hospital discharge reports (HDR). This document must contain a minimum data set, and should be understandable to the patient, family doctor or other professionals. The acquisition of this competence has been little studied, and the quality of HDRs performed by MIR has not been previously evaluated.

Subjects and methods. The present study evaluated the quality of 124 emergency HDRs comparing those signed by specialists, only signed or signed by MIR, or signed by MIR and specialist in the fields of Orthopaedic Surgery and General Surgery.

Results. The result has been more favourable than that of previous publications, with a score of 7 out of 10 in $94.4 \%$ of cases. HDRs made by first-year residents and supervised by specialists obtained the best scores with statistically significant differences $(p=0.003)$. No differences were observed between the reports by the morning or night $(p=0.6)$. In terms of content, $12.9 \%$ of reports had acronyms difficult to understand.

Conclusion. Our study depicts the beneficial effect of HDR monitoring in the first year of residency as well as an improvement objective by minimizing the use of medical abbreviations.

Key words. Hospital discharge report. MIR. Postgraduate medical education.

\section{Introducción}

La historia clínica electrónica es actualmente una pieza clave en la atención sanitaria. Es esencial una adecuada formación para poder acceder a la información que contiene e interpretarla, registrar datos y generar documentos. Esta formación debería ini- ciarse en pregrado [1] y se completaría durante la formación sanitaria especializada [2]. El médico interno residente (MIR) debe ser competente en la redacción de un adecuado informe de alta hospitalaria (IAH) [3]; debe cumplir con unos contenidos mínimos, y el contenido ha de ser comprensible por el propio paciente pero también por los familiares $\mathrm{u}$
Servicio de Cirugía Ortopédica y Traumatología (J.A. FernándezValencia, M. Ríos, J. Riba); Servicio de Documentación Clínica (A. Conesa); Servicio de Cirugía General y Digestiva (X. Morales); Hospital Clínic. Consorci d'Atenció Primària de Salut de l'Eixample (M. Catalán). Barcelona, España.

Correspondencia:

Dr. Jenaro A. Fernández-Valencia. Unidad de Cadera. Servicio de Cirugía Ortopédica y Traumatología. Hospital Clínic. Universitat de Barcelona. Villarroel, 170 E-08036 Barcelona.

E-mail: jenarofv@clinic.ub.es Conflicto de intereses: No declarado.

Competing interests: None declared.

(c) 2015 FEM 
Tabla I. Sistema de puntuación de los informes de alta hospitalaria (puntuación IAH).

\begin{tabular}{|c|c|}
\hline \multirow{4}{*}{ Anamnesis } & $\begin{array}{l}\text { 3. Completa (alergias, antecedentes } \\
\text { y enfermedad actual) }\end{array}$ \\
\hline & 2. Bastante completa (falta alguna anotación) \\
\hline & 1. Incompleta (falta alguno de los apartados) \\
\hline & 0. No consta \\
\hline \multirow{4}{*}{ Exploración física } & $\begin{array}{l}\text { 3. Completa (inspección general, circulatorio, } \\
\text { respiratorio, abdomen y neurológica) }\end{array}$ \\
\hline & $\begin{array}{l}\text { 2. Bastante completa (aparato/sistema } \\
\text { motivo de consulta y otros, pero no todos) }\end{array}$ \\
\hline & $\begin{array}{l}\text { 1. Incompleta (falta la exploración } \\
\text { de la mayoría de aparatos/sistemas) }\end{array}$ \\
\hline & 0. No consta \\
\hline \multirow{2}{*}{$\begin{array}{l}\text { Resultados de técnicas } \\
\text { y exploraciones } \\
\text { complementarias }\end{array}$} & 1. Consta \\
\hline & 0. No consta \\
\hline \multirow{2}{*}{$\begin{array}{l}\text { Orientación } \\
\text { diagnóstica }\end{array}$} & 1. Consta \\
\hline & 0. No consta \\
\hline \multirow{2}{*}{ Plan terapéutico } & 1. Consta \\
\hline & 0. No consta \\
\hline \multirow{2}{*}{ Seguimiento al alta } & 1. Consta \\
\hline & 0. No consta \\
\hline
\end{tabular}

otros profesionales como asistentes sociales, fisioterapeutas, documentalistas clínicos, abogados o jueces. Los tutores debemos proporcionar feedback sobre esta actividad y se plantean las siguientes preguntas: ¿cómo evaluar la calidad de los IAH?, ¿en qué momento el MIR puede realizar un IAH sin supervisión? La evaluación de los registros generados por MIR en la historia clínica electrónica podría ser de utilidad en la detección de áreas de mejora [4]. La revisión de la bibliografía en cuanto a formación en esta competencia muestra escasas referencias [2]. Así mismo, existen pocas publicaciones en nuestro país sobre la calidad de los informes de alta en general y, más concretamente, en relación con la formación especializada.

El objetivo del presente estudio es determinar la calidad del IAH de un servicio de urgencias, realizado por residentes de especialidades quirúrgicas en un hospital de tercer nivel, con el objetivo de determinar si la calidad del informe tiene correlación con el progreso de la residencia y también para detectar posibles recomendaciones de mejora.

\section{Sujetos y métodos}

Estudio descriptivo transversal en el que se evaluó la calidad de los IAH de urgencias cumplimentados por MIR de dos especialidades quirúrgicas: cirugía general y traumatología y cirugía ortopédica.

El servicio de urgencias pertenece a un hospital universitario de tercer nivel, con 850 camas de hospitalización, que cubre la atención médica urgente de una población urbana de unas 500.000 personas. El periodo estudiado comprendió desde el 1 de julio de 2012 al 30 de junio de 2013. Se seleccionó una muestra de IAH de dicho periodo, de forma aleatoria. Se incluyeron en el análisis todos los IAH firmados por residentes, incluyendo los firmados por residentes de primer año bajo supervisión por un especialista. También se incluyeron en el estudio los informes generados por los especialistas para ser tomados como patrón de referencia.

La evaluación de la calidad de los informes de alta se basó en un sistema de puntuación de uso interno por la Comisión de Historias Clínicas en nuestro centro, adaptado para este estudio, que denominamos 'puntuación IAH' (Tabla I), la cual proporciona un valor mínimo de 0 y un valor máximo de 10 puntos. Para la obtención de esta puntuación, se estudió la presencia de los apartados de obligado cumplimiento: anamnesis (0-3 puntos), exploración física (0-3 puntos), resultados de técnicas/exploraciones complementarias (0-1 punto), orientación diagnóstica (0-1 punto), plan terapéutico (0-1 punto) y seguimiento al alta (0-1 punto). Dicha evaluación fue realizada por dos tutores de médicos internos residentes (J.A.F.V. y M.R.). Se consideraron informes de calidad insuficiente aquellos con valor inferior a 7. En cuanto a este nivel de corte, es importante tener en cuenta que en el contexto de urgencias, la gravedad de los casos y la sobrecarga asistencial puede justificar una mayor síntesis de la anamnesis, pudiendo considerarse que un informe es correcto con dos de los tres puntos en este apartado. Del mismo modo, en relación con la exploración física, podría estar justificado en aras de una mayor eficiencia limitarla al sistema que motiva la consulta urgente en casos como un esguince de tobillo o una herida incisa en antebrazo y, en consecuencia, este apartado podría ser evaluado disminuyendo dos puntos su valor máximo. Por ello, en el presente estudio úni- 
camente se han considerado los IAH como insuficientes cuando puntuaron menos de 7 .

Por otro lado, se realizó una evaluación por un especialista en medicina familiar y comunitaria (M.C.), quien evaluó el grado de inteligibilidad del informe en base a una clasificación previamente descrita por Conesa et al [5] (Tabla II), la presencia o ausencia de siglas poco usuales o de difícil comprensión (para el médico de atención primaria o para el propio paciente), y la presencia o ausencia de contenido redundante (p. ej., el volcado completo de resultados analíticos o de informes de pruebas de imagen que no aportan información relevante).

El tamaño de la muestra se determinó a priori, utilizando el programa online del Institut de Recerca de l'Hospital del Mar, GRANMO 7.12 [6]. Aceptando un riesgo $\alpha$ de 0,05 y un riesgo $\beta$ de 0,2 en un contraste bilateral, se calcularon un mínimo de 19 sujetos en el grupo 1 y un mínimo de 76 en el grupo 2 para detectar como estadísticamente significativa la diferencia entre dos proporciones, esperando diferencias de un $40 \%$ entre ambos grupos. Dado que para el periodo estudiado se disponía de historia clínica electrónica, se esperó una accesibilidad al $100 \%$ de los informes seleccionados por muestreo. De esta forma se determinó como necesaria una muestra mínima de 95 IAH.

\section{Selección de informes de alta a evaluar}

El sistema de muestreo fue aleatorio estratificado. Mediante una secuencia generada por ordenador, se eligieron al azar tres meses del año, y de cada mes, cinco días al azar. Introducidas las variables en el programa Randomizer (disponible en www.random.org), se determinó que los meses a evaluar serían septiembre de 2012, diciembre de 2012 y enero de 2013. Se evaluaron los informes de alta de los primeros pacientes atendidos a partir de las 10:00 $\mathrm{h}$ en cirugía ortopédica y traumatología (dos pacientes) y en cirugía general (dos pacientes) para cada uno de estos tres meses: los días del mes de septiembre fueron el 9, 19, 21, 23 y 28; los de diciembre, el 4, 17, 19, 22 y 29, y los de enero, el 6, 11, 13, 17 y 24. Por otro lado, se evaluaron los primeros pacientes atendidos a partir de las 03:00 h en cirugía ortopédica y traumatología (dos pacientes) y en cirugía general (dos pacientes), para cada uno de estos tres meses: los días del mes de septiembre fueron el 2, 6, 19, 25 y 30; los de diciembre, el 2, 7, $11,18,23$ y 30, y los de enero, el 1, 7, 10, 21 y 28. Esto proporcionó un total de 124 IAH evaluados. En ninguno de los casos el paciente falleció en urgencias y en nueve casos el IAH de urgencias fue
Figura. Número de informes de alta hospitalaria realizado por cada grupo: médico interno residente (MIR), MIR con especialista o especialista. MIR1 + E: MIR de primer año y especialista; MIR2: MIR de segundo año; MIR2 + E: MIR de segundo año y especialista; MIR3: MIR de tercer año; MIR3 + E: MIR de tercer año y especialista; MIR4: MIR de cuarto año; MIR5: MIR de quinto año; MIR5 + E: MIR de quinto año + E; E: especialista



Tabla II. Tipo de informe de alta hospitalaria.

Tipo 1 Informes legibles, bien estructurados y con toda la información necesaria

Tipo 2 Informes poco estructurados, moderadamente difíciles de leer, en los que es posible identificar diagnósticos y procedimientos, con estructura mejorable, aunque con información suficiente

Tipo 3 Informes mal redactados, difíciles de leer con normalidad, sin un diagnóstico bien definido por escrito, no estructurados y con escasa información

para ingreso en el propio hospital. La distribución del número de IAH por cada grupo estudiado se muestra en la figura.

El proceso de registro de la información y su evaluación se rigió por lo dispuesto en la Ley Orgánica 15/1999 de Protección de Datos de Carácter Personal.

\section{Análisis estadístico}

El programa estadístico utilizado para el estudio fue SOFA Statistics. En base a la prueba de KolmogorovSmirnov se determinó que las puntuaciones obtenidas en IAH presentaban una distribución normal. Los tests paramétricos para el contraste de hipótesis fueron los siguientes:

- Para determinar si existían diferencias significativas en las puntuaciones IAH entre informes realizados por MIR de primer año y el resto se utilizó la $t$ de Student, con una significación de $p<0,05$. Se utilizó el mismo test para determinar si existían diferencias significativas en las puntuacio- 
nes IAH entre informes realizados por la mañana (a partir de las 10:00 h) e informes realizados de madrugada (a partir de las 03:00 h).

- Para determinar si existían diferencias en la tipología de informe de alta, la presencia de siglas incompresibles o contenido redundante, se utilizó un test de chi al cuadrado para cada grupo respecto de la variable estudiada, con una significación de $p<0,05$.

\section{Resultados}

La puntuación media de la calidad del IAH de los MIR de primer año supervisados por especialista fue de 8,80 \pm 0,72 (rango: 7-10), mientras que el resto de informes obtuvieron una puntuación media de 8,2 $\pm 1,2$ (rango: 4-10). Estas diferencias fueron estadísticamente significativas $(p=0,003)$.

Sólo siete informes obtuvieron una puntuación IAH menor de 7: un informe puntuó 4, cuatro puntuaron 5, y dos puntuaron 6 . El resto de informes puntuaron con valor 7 o superior: 11 informes puntuaron 7, 41 informes puntuaron 8 , nueve informes puntuaron 54, y 11 informes puntuaron 10. De esta forma, el 5,64\% de los informes evaluados se consideraron con calidad insuficiente.

La media del IAH obtenida por los informes firmados únicamente por MIR fue de 8,1 $\pm 1,3$ (rango: 4-10), mientras que la media de los IAH firmados por especialistas o por MIR con especialista fue de 8,692 $\pm 0,7$ (rango: 7-10), siendo estas diferencias estadísticamente significativas $(p=0,005)$.

En el grupo de informes de pacientes atendidos a partir de las 10:00 h, la media de puntuación IAH fue de 8,4 $\pm 1,1$ (rango: 5-10), mientras que la media en el grupo de pacientes atendido a partir de las 03:00 $\mathrm{h}$ fue de 8,3 $\pm 1,1$ (rango: 4-10), sin mostrar diferencias estadísticamente significativas $(p=0,605)$.

En cuanto a comprensión, los IAH fueron legibles y bien estructurados (tipo 1) en 118 casos (95\%) y poco estructurados o moderadamente difíciles de leer (tipo 2) en seis casos (5\%), sin observarse informes difíciles de leer (tipo 3). Respecto al uso de siglas, el $12,9 \%$ de los IAH presentaba siglas consideradas poco usuales o de difícil comprensión para el médico de atención primaria o para el propio paciente. Únicamente uno de los IAH firmado por un MIR y especialista las tenía, mientras que 15 IAH del resto presentaba estas siglas poco usuales o de difícil comprensión $(p=0,048)$.

Respecto a la presencia de contenido redundante, sólo en tres casos se observó contenido redundante, que no afectaba a la compresión de los IAH.

\section{Discusión}

El Real Decreto 183/2008, artículo 15, referente a la responsabilidad progresiva del residente, indica que los informes realizados por MIR de primer año deben ser supervisados y firmados por especialistas. Hasta la fecha, ningún estudio había evaluado el resultado de esta supervisión. El presente estudio muestra que los informes de alta realizados por MIR de primer año y supervisados por especialistas fueron los de mayor calidad, con diferencias estadísticamente significativas respecto a los informes realizados por el resto de MIR o especialistas durante el mismo periodo.

A pesar de que el estudio no puede garantizar que un informe únicamente firmado por MIR no tuviera supervisión por especialista (una de las limitaciones del estudio), es llamativo que informes que fueron firmados sólo por MIR obtuvieron peor puntuación que los firmados conjuntamente con un especialista, de forma estadísticamente significativa.

Determinar el momento en que el residente puede realizar un informe de alta sin supervisión dependerá del proceso de adquisición de esta competencia y de la evaluación de este proceso por parte de aquel que supervisa la actividad del residente. De manera generalizada, aunque el aprendizaje para la realización de un IAH se inicia durante la formación de pregrado, los primeros años de residencia son esenciales para la adquisición de esta competencia [7]. Por un lado, en este proceso de aprendizaje influyen factores como el estilo general y las costumbres del centro donde ejerce el MIR, pero, por otro, la calidad de los informes dependerá en gran manera de que la enseñanza acerca de su elaboración sea estructurada y supervisada, ya que una vez establecido el estilo, será difícil modificarlo. Los registros en la historia clínica electrónica constituyen una excelente herramienta para proporcionar feedback docente [8] y proponemos utilizar la puntuación IAH para evaluar la calidad de los IAH de nuestros MIR, pudiendo adaptarse a las necesidades formativas en cada contexto. Sin embargo, este estudio no ha considerado otros aspectos tales como la adecuación del diagnóstico, la adecuación del tratamiento en relación al diagnóstico u otras muchas cuestiones relativas a la calidad intrínseca de la atención médica proporcionada.

La calidad del IAH fue satisfactoria en el 94,4\% de los 124 informes evaluados. Esta puntuación se encuentra por encima de la obtenida en publicaciones previas que evaluaban la calidad de informes de alta sin diferenciar si estaban firmados o no por MIR. Sardá et al [9] describieron que la cumplimentación 
de los distintos apartados de los informes de alta de su centro varió del $90 \%$ para el diagnóstico y motivo de ingreso hasta poco más del $50 \%$ para el plan de seguimiento y el tratamiento. En este estudio, la cumplimentación fue superior en los servicios médicos que en los quirúrgicos para casi todos los apartados analizados. En una encuesta a médicos generales y especialistas incluida en el mismo estudio, la mayoría de facultativos en ambos grupos preferían informes cortos y resumidos que largos y detallados. Zambrana-García et al referían que sólo el $58 \%$ de los informes de alta en servicios de medicina interna de 24 hospitales andaluces contenían datos clínicos y administrativos suficientes y que la calidad de los informes era mayor en los hospitales más pequeños [10]. Conesa et al determinaron en un estudio multicéntrico que un 9-20\% de los informes de alta de urgencias presentaban un contenido informativo insuficiente o eran prácticamente ilegibles [5]. Gómez-Rosado et al publicaron una revisión de informes de alta de un servicio de cirugía, indicando que sólo el $41 \%$ eran válidos, y el resto, insuficientes o claramente no válidos [11].

Creemos remarcable que los residentes de segundo y tercer año tendiesen a acumular la mayor parte de los IAH puntuados como de menor calidad y, sin embargo, los residentes de segundo y tercer año que firmaron conjuntamente con un especialista obtuvieran puntuaciones por encima de 7 en todos los casos. Consideramos que estas diferencias ilustran el efecto beneficioso de la supervisión y, por otro lado, que la calidad del IAH sin supervisión a partir del segundo año de residencia disminuye ligeramente, aunque puede considerarse como aceptable.

Respecto a la presencia de siglas de difícil comprensión para el médico de familia, el 12,9\% detectado podría ser criticable, dependiendo del contexto. Algunas de estas siglas fueron: FX por fractura, MTT por metatarsiano, PC por paciente, HDE por hemodinámicamente estable, SRIS por síndrome de respuesta inflamatoria sistémica y HCD por hipocondrio derecho. Las aceptadas por uso común en este estudio fueron siglas como HTA por hipertensión arterial, DLP por dislipemia, VAT por vacuna antitetánica, PPLB por puño percusión lumbar bilateral, FII por fosa ilíaca izquierda, FID por fosa ilíaca derecha o TCE por traumatismo craneoencefálico. Consideramos que, en lo posible, debe minimizarse el uso de siglas para facilitar la comprensión de los IAH por todos sus lectores potenciales.

La principal limitación del estudio se encuentra en la supervisión no registrada del informe de alta. Cada IAH no firmado por especialista puede haber tenido su supervisión. Sin embargo, en base a la práctica habitual en nuestro centro, podemos asumir que si bien el proceso asistencial es supervisado por los especialistas (con supervisión decreciente a lo largo de la residencia), el informe de alta de urgencias que se entrega al paciente en la mayoría de ocasiones no tiene esa supervisión en cuanto a su contenido y estilo de redacción a partir del segundo año de residencia, salvo que exista la firma del especialista en el informe.

El presente estudio evalúa cumplimentación de diferentes apartados en el documento de alta, pero no otros criterios de calidad intrínseca del proceso, como la adecuación del diagnóstico, la adecuación del tratamiento al diagnóstico o la adhesión a guías de tratamiento del propio centro.

Otra limitación consiste en la representatividad de la muestra, ya que únicamente se evalúan dos especialidades y los resultados no pueden extrapolarse al total de MIR de especialidades quirúrgicas de nuestro centro.

En conclusión, el presente estudio ha permitido obtener datos sobre la calidad de los IAH de urgencias realizados por los MIR de dos especialidades quirúrgicas en nuestro centro. El resultado ha sido más favorable que el de publicaciones previas, con una correcta cumplimentación en el 94,4\% de los casos, sin que se hayan encontrado diferencias entre informes realizados por la mañana o por la madrugada. Además, los IAH realizados por residentes de primer año y supervisados por especialistas han sido los informes con mejor puntuación. En cuanto a los contenidos, se debería trabajar para minimizar el uso de siglas, ya que si bien en el presente estudio el $12,9 \%$ no eran comprensibles por el médico de familia, sería previsible que los pacientes no comprendieran buena parte de las siglas que actualmente consideramos de uso común.

\section{Bibliografía}

1. Stephens MB, Williams PM. Teaching principles of practice management and electronic medical record clinical documentation to third-year medical students. J Med Pract Manage 2010; 25: 222-5.

2. Stephens MB, Gimbel RW, Pangaro L. Commentary: the RIME/EMR scheme -an educational approach to clinical documentation in electronic medical records. Acad Med 2011; 86: 11-4.

3. Von Fragstein M, Silverman J, Cushing A, Quilligan S, Salisbury H, Wiskin C; UK Council for Clinical Communication Skills Teaching in Undergraduate Medical Education. UK consensus statement on the content of communication curricula in undergraduate medical education. Med Educ 2008; 42: 1100-7.

4. Ricarte-Díez JI, Martínez-Carretero JM. Formación del residente desde su abordaje como adulto. Educ Med 2008; 11: 131-8. 
5. Conesa A, Muñoz R, Torre P, Gelabert G, Casanellas JM, Trilla A, Asenjo MA. Evaluación de los informes de asistencia de urgencias como instrumento de gestión de la información clínica. Med Clin (Barc) 2003; 120: 734-6.

6. Institut de Recerca de l'Hospital del Mar. Programa de cálculo de tamaño de la muestra, GRANMO 7.12. URL: http://www. imim.cat/ofertadeserveis/software-public/granmo/. [07.12.2012].

7. García-Alegría J. La calidad de los informes clínicos. URL http://www.calidadyriesgo.es/calidadasistencial/25-dr-d-josejavier-garcia-alegria. [15.01.2013].

8. Seifan A, Mandigo M, Price R, Galetta S, Jozefowicz R, Jaffer A, et al. Education research: can my electronic health record teach me something? A multi-institutional pilot study. Neurology 2013; 80: e98-103.
9. Sardá N, Vilá R, Mira M, Canela M, Jariod M, Masqué J. Análisis de la calidad y contenido del informe de alta hospitalaria. Med Clin (Bar) 1993; 101: 241-4.

10. Zambrana-García JL, Rivas-Ruiz F; en nombre del Grupo para el Análisis de la Calidad del Informe de Alta Hospitalaria en los Hospitales de Alta Resolución de Andalucía. Calidad de los informes de alta hospitalaria respecto a la legislación vigente y las recomendaciones consensuadas por expertos. Gac Sanit 2013; 27: 450-3.

11. Gómez-Rosado JC, Sánchez-Ramírez M, Valdés-Hernández J, Capitán-Morales LC, Del Nozal-Nalda MI, Oliva-Mompeán F. Importance of the quality of the discharge report in the management of a surgical clinical unit. Cir Esp 2013; 91 : 378-83. 